\title{
3 Research Square

\section{Robot-Assisted vs. Laparoscopic Radical Prostatectomy for Immediate- and High-Risk Localized Prostate Cancer: A Propensity-Score Matched Analysis}

\section{Deng Wen}

First Affiliated Hospital of Nanchang University

\section{Zhang Cheng}

First Affiliated Hospital of Nanchang University

Jiang Hao

First Affiliated Hospital of Nanchang University

\section{Li Yulei}

First Affiliated Hospital of Nanchang University

\section{Liu Xiaoqiang}

First Affiliated Hospital of Nanchang University

Chen Luyao

First Affiliated Hospital of Nanchang University

\section{Liu Weipeng}

First Affiliated Hospital of Nanchang University

\section{Zhou Xiaochen}

First Affiliated Hospital of Nanchang University

\section{Sun Ting}

First Affiliated Hospital of Nanchang University

\section{Wang Gongxian}

First Affiliated Hospital of Nanchang University

Bin Fu ( $\square$ urofbin@163.com )

First Affiliated Hospital of Nanchang University

\section{Research}

Keywords: radical prostatectomy, robot, laparoscopic, immediate-risk, high-risk, prostate cancer.

Posted Date: May 7th, 2021

DOI: https://doi.org/10.21203/rs.3.rs-442704/v1 
License: (c) (i) This work is licensed under a Creative Commons Attribution 4.0 International License. Read Full License 


\section{Abstract \\ Background}

To evaluate the functional and oncological efficacy of robot-assisted radical prostatectomy (RARP) and laparoscopic radical prostatectomy (LRP) for immediate- and high-risk localized prostate cancer (PCa).

\section{Methods}

232 patients bearing immediate- and high-risk localized PCa between January 2016 and October 2019 were enrolled according to the inclusion criteria. The perioperative, functional and oncological outcomes were compared between the RARP and LRP groups after applying the propensity-score matching (PM) (1:1) method, which were employed to attenuate the impact of the potential baseline confounders.

\section{Results}

In all, except for 10 patients without a suitable pair, the remaining patients in the LRP group were successfully matched to 85 patients in the RARP arm. All differences in preoperative variables turned to be insignificant after PM. Within the matched cohort, no open conversion was required in both groups. The RARP group was corrected with a significantly shorter mean operative time than the LRP group ( $p<$ 0.001). Patients in the RARP arm were also at a lower risk of $\leq$ Grade Il complications than those in the LRP group $(p=0.036)$. Meanwhile, the proportions of transfusion and $\geq$ Grade II complications in the RARP group were similar to that in the LRP group ( $p=0.192$ and $p=1.000$, respectively). No significant differences regarding the mean estimated blood loss, rates of pT3 disease and positive surgical margin, median specimen Gleason score and hospital stay length existed between the two groups. RARP vs. LRP tended to a significantly higher percentage of urinary continence at the removal of catheter $(p=0.031)$, postoperative 6 months $(p=0.043)$, and last follow-up $(p=0.046)$. Significant differences were also found between the RARP and LRP arms in erectile function at postoperative 6 months and last follow-up $(p=0.013$ and $p=0.009$, respectively). The statistical comparability between the two groups was observed in biochemical recurrence-free survival $(p=0.228)$.

\section{Conclusions}

For surgically managing immediate- and high-risk localized PCa, RARP tended to a lower risk of $\leq$ Grade II complications and superior functional preservation without cancer control being compromised when comparing with LRP.

\section{Background}


Prostate cancer ( $\mathrm{PCa})$, the second most common cancer and the fifth dominating cause of cancerspecific mortality among men around the world (1), is increasingly been discovered due to the widespread diffusion of prostate specific antigen (PSA) screening, markedly the localized ones $(2,3)$. Given the slowly evolving nature of localized prostate tumors, it is of great significance to distinguish PCa destined to cause clinical symptoms or metastases from more clinically indolent PCa that is highly unlikely to impact survival to reduce overtreatment in patients with PCa without compromising opportunities for cure. The D'Amico risk classifications proposed on the basis of clinical and pathological characteristics including the clinical stage, PSA, and biopsy Gleason score have been widely endorsed worldwide (3). The role of radical prostatectomy (RP) in managing D'Amico low-risk PCa is in doubt due to the similar survival benefit of patients with low-risk localized PCa following immediate radical treatment and active surveillance and RP-related harms to quality of life (3-5), whilst RP has a tendency towards a better survival benefit over watchful waiting for immediate-risk localized PCa $(4,6,7)$ and 10-year PCa-specific survival rates after RP coupled with node dissection were generally over $90 \%$ and remarkably consistent across all large studies focusing on high-risk PCa (8). In 2020, surgery is not recommended in those patients with low-grade, low-volume Gleason $6 \mathrm{PCa}$ in consideration of the little clinical benefit and considerable adverse effects following surgery, while RP is considered appropriate for men with intermediate- and high-risk disease (9).

With the superiority of surgical robots in three-dimensional magnified vision of the surgical field, improved dexterity, and higher precision during the surgical procedure, robot-assisted RP (RARP) is considered as a great evolution of minimally invasive surgery to reduce the difficulty associated with complex laparoscopic surgery (10) and has been widely disseminated for localized PCa since 2001 (11). However, thus far, whether the advantages of RARP over laparoscopic RP (LRP) mentioned above could translate into superior functional preservation and oncological control was still inconclusive due to the scarcity of high-level evidence comparing RARP and LRP for localized PCa $(10,12)$. There are only three randomized controlled trials (RCTs) concentrating on comparing RARP and LRP for localized PCa with different endpoints over the short-term study periods (13-15), which is far from reaching a convincing consensus about the controversy. What's worse, no study focusing on comparing RARP and LRP for patients with immediate- and high-risk localized PCa has been reported yet, while the cogent evidence comparing the efficacy and safety of RARP and LRP for immediate- and high-risk localized PCa is of great clinical importance.

To close this gap of cogent evidence concerning the functional and oncological efficacy of RARP and LRP for immediate- and high-risk localized PCa, we designed this first analysis comparing RARP and LRP for immediate- and high-risk localized PCa in an attempt to document differences in the perioperative, functional and oncological outcomes obtained after the two techniques in a retrospective fashion.

\section{Methods}

Having obtained the approval of the Ethnic Committee of the First Affiliated Hospital of Nanchang University, we meticulously reviewed our prospectively maintained database to retrospectively gather all 
the demographic, clinical, and pathologic information of patients harbouring localized PCa between January 2016 and October 2019. All patients with PCa were screened and incorporated into the final analysis on the grounds of the following eligibility criteria: (1) total serum PSA $\geq 10 \mathrm{ng} / \mathrm{mL}$ or Gleason score $\geq 7$ or localized T2b or T2c stage; (2) patients undergoing RARP or LRP for localized PCa; (3) no evidence of clinical positive lymph nodes or clinical T3-4 stage. Only when the case simultaneously satisfied all these inclusion criteria were the men included, whilst the others were excluded from our study. On the basis of inclusion criteria described above, included in the final analysis were 232 patients, of whom 137 and 95 patients were divided by surgical approaches into the RARP and LRP arms, respectively, and none of them had a history of abdominal surgery. Prostate magnetic resonance imaging and bone scintigraphy were routinely performed in all patients before surgeries.

All surgeries were conducted with the anterior approach by three highly experienced hands (Fu B, Wang GX, and Sun T). Prior to this study initiation, each of these three surgeons had performed more than 300 LRPs and 100 RARPs as an operator or a trainee. The patients' assignment was usually at the discretion of these four highly experienced surgeons according to tumor and patient characteristics. The written informed consent was acquired from each patient in both groups. The anterior approach to RARP was done according to the techniques established by Menon et al. (16), while the anterior approach to LRP was completed following the surgical steps described by Touijer et al. (17). Pelvic lymph node dissection (PLND) was routinely done in all high-risk patients and those immediate-risk cases with a preoperative estimated risk exceeding $5 \%$ in nodal involvement, while the nodal dissection could be omitted at a low risk of missing positive nodes in other immediate-risk men. A standardized extended PLND (ePLND) template with the upper margin being the common iliac artery was closely observed when performing all these lymph node dissections. Nerve sparing was preoperatively proposed according to clinical features and intraoperatively modified based on evidence of bundle invasion.

Baseline demographics and clinical elements (age, body mass index [BMI], diabetes mellitus, hypertension, American Society of Anesthesiologists [ASA] score, preoperative total PSA, preoperative erectile function quantified in accordance with the International Index of Erectile Function [IIEF]-5 score (18), and risk stratification assessed with D'Amico risk classifications (3)) and preoperative tumor characteristics (clinical TNM stage, biopsy Gleason score, and prostate volume calculated by the virtue of transrectal ultrasound) were extracted from the database.

Data with regard to perioperative outcomes (operative time [OT], estimated blood loss [EBL], ePLND, nerve sparing procedure, open conversion, transfusion, postoperative hospital stay, and postoperative complications evaluated with Clavien-Dindo classification (19)) and pathologic results (pathological T stage, specimen Gleason score, positive surgical margin [PSM] which were defined as tumor extending to the inked-surface of prostate specimen, and positive lymph node) was also retrieved from our database. Information about postoperative complications could also be collected via chart reviews or face-to-face and telephone interviews. 
The median postoperative follow-up length for the RARP and LRP groups was 29 and 23 months, respectively. Postoperative follow-up was regularly arranged every 3 months within the first year after surgery and every 6 months since the second year after surgery for each patient. Each case routinely underwent postoperative PSA tests every 3 months to monitor biochemical recurrence (BCR), which was considered as the occurrence after prostatectomy that two consecutive rising serum PSA measured on two separate occasions was $0.2 \mathrm{ng} / \mathrm{mL}$ or greater. UC was defined by the use of $\leq$ one dry pad over the 24-hour period. The total PSA level and erectile function score were reported at postoperative 6 months and last follow-up, while the rate of UC recovery were presented at the removal of catheter, postoperative 6 months, and last follow-up.

Propensity-score matching (PM) method was employed to impose restrictions on significant differences in preoperative clinical and tumor characteristics. The propensity score was calculated with nonparsimonious multivariate logistic regression on the basis of all preoperative variables, namely, age, BMI, diabetes mellitus, hypertension, ASA score, preoperative total PSA, preoperative IIEF- 5 score, clinical TNM stage, biopsy Gleason score, and prostate volume. Finally, except for 10 patients missing an appropriate pair, all the remaining cases in the LRP arm were successfully matched to 85 patients in the RARP group using a 1:1 ratio with the nearest neighbour pairing method. All differences before and after PM in preoperative elements were analyzed between the RARP and LRP arms with the independent t-test or the Pearson chi-squared test being utilized.

All non-normally distributed continuous variables were presented as median and interquartile range and compared using the Wilcoxon rank-sum test, whilst those in a normally distributed fashion were presented as mean and standard deviation and analyzed with the independent t-test. All categorical variables were presented as proportion and percentages and calculated with the Pearson chi-squared test or the Fisher's exact test being used. The Kaplan-Meier method was employed to estimate BCR-free survival probabilities and the proportions of postoperative return to UC with the log-rank test. All statistical analyses were conducted on STATA version 12.0 (STATA corp., College Station, TX), and the statistical significance was defined a two-sided $p$ value of $<0.05$.

\section{Results}

All preoperative information concerning the clinical and tumor characteristics before and after PM was detailedly described in Table 1. In all, 232 patients with primary immediate- and high-risk localized PCa in line with the inclusion criteria, comprising 137 RARPs and 95 LRPs, were enrolled in this analysis over the study period being reviewed. Before the PM, the RARP group was significantly corrected with a lower mean age (65.4 vs. 68.0 years, $p=0.010)$, higher mean BMI ( 23.2 vs. $\left.22.2 \mathrm{~kg} / \mathrm{m}^{2}, p=0.044\right)$, smaller mean prostate volume (38.4 vs. $43.9 \mathrm{~mL}, p=0.001)$, higher median preoperative IIEF-5 score (17 vs. $15, p$ $=0.011$ ), and lower median biopsy Gleason score ( 6 vs. $7, p=0.020)$ than the LRP group, while no significant differences in other preoperative variables existed between the two arms. All these significant differences in preoperative variables disappeared within the well-balanced matched cohorts after applying the PM method (Table 1). 
Table 1

Preoperative characteristics by surgery type before and after propensity score matching.

\begin{tabular}{|c|c|c|c|c|c|c|}
\hline \multirow[t]{2}{*}{ Variable } & \multicolumn{3}{|c|}{$\begin{array}{l}\text { Before propensity score } \\
\text { matching }\end{array}$} & \multicolumn{3}{|c|}{$\begin{array}{l}\text { After propensity score } \\
\text { matching }\end{array}$} \\
\hline & $\begin{array}{l}\operatorname{RARP}(n= \\
137)\end{array}$ & $\begin{array}{l}\operatorname{LRP}(n \\
=95)\end{array}$ & $\begin{array}{l}p \\
\text { value }\end{array}$ & $\begin{array}{l}\text { RARP }(n \\
=85)\end{array}$ & $\begin{array}{l}\operatorname{LRP}(n= \\
85)\end{array}$ & $\begin{array}{l}p \\
\text { value }\end{array}$ \\
\hline Age, years, mean (SD) & $65.4(7.3)$ & $\begin{array}{l}68.0 \\
(7.3)\end{array}$ & 0.010 & $65.5(7.3)$ & $67.2(7.2)$ & 0.138 \\
\hline BMI, kg/m2, mean (SD) & $23.2(3.5)$ & $\begin{array}{l}22.2 \\
(3.8)\end{array}$ & 0.044 & $23.1(3.6)$ & $22.7(3.8)$ & 0.482 \\
\hline Diabetes mellitus (yes), n (\%) & $17(13.4 \%)$ & $\begin{array}{l}15 \\
(15.8 \%)\end{array}$ & 0.614 & $\begin{array}{l}12 \\
(14.1 \%)\end{array}$ & $\begin{array}{l}14 \\
(16.5 \%)\end{array}$ & 0.670 \\
\hline Hypertension (yes), n (\%) & $33(26.0 \%)$ & $\begin{array}{l}23 \\
(24.2 \%)\end{array}$ & 0.763 & $\begin{array}{l}22 \\
(25.9 \%)\end{array}$ & $\begin{array}{l}20 \\
(23.5 \%)\end{array}$ & 0.722 \\
\hline ASA score $(\geq 3), n(\%)$ & $7(5.5 \%)$ & $7(7.4 \%)$ & 0.573 & $4(4.7 \%)$ & $5(5.9 \%)$ & 1.000 \\
\hline $\begin{array}{l}\text { Preoperative total PSA, } \mathrm{ng} / \mathrm{mL} \text {, } \\
\text { mean (SD) }\end{array}$ & $25.7(25.5)$ & $\begin{array}{l}26.6 \\
(26.9)\end{array}$ & 0.795 & $\begin{array}{l}27.5 \\
(27.9)\end{array}$ & $\begin{array}{l}26.6 \\
(28.1)\end{array}$ & 0.834 \\
\hline $\begin{array}{l}\text { Prostate volume, } \mathrm{mL} \text {, mean } \\
\text { (SD) }\end{array}$ & $38.4(10.6)$ & $\begin{array}{l}43.9 \\
(13.0)\end{array}$ & 0.001 & $\begin{array}{l}39.7 \\
(10.8)\end{array}$ & $\begin{array}{l}42.8 \\
(13.0)\end{array}$ & 0.093 \\
\hline $\begin{array}{l}\text { Preoperative IIEF-5 score, } \\
\text { median (IQR) }\end{array}$ & $17(14,19)$ & $\begin{array}{l}15(13 \\
18)\end{array}$ & 0.011 & $\begin{array}{l}18(14.3 \\
19)\end{array}$ & $\begin{array}{l}16(13 \\
18.75)\end{array}$ & 0.113 \\
\hline cTNM stage, n (\%) & & & 0.365 & & & 0.385 \\
\hline T1-T2a & $52(41.0 \%)$ & $\begin{array}{l}48 \\
(50.5 \%)\end{array}$ & & $\begin{array}{l}37 \\
(43.5 \%)\end{array}$ & $\begin{array}{l}46 \\
(54.1 \%)\end{array}$ & \\
\hline $\mathrm{T} 2 \mathrm{~b}$ & $46(36.2 \%)$ & $\begin{array}{l}29 \\
(30.5 \%)\end{array}$ & & $\begin{array}{l}31 \\
(36.5 \%)\end{array}$ & $\begin{array}{l}25 \\
(29.4 \%)\end{array}$ & \\
\hline $\mathrm{T} 2 \mathrm{c}$ & $29(22.8 \%)$ & $\begin{array}{l}18 \\
(19.0 \%)\end{array}$ & & $\begin{array}{l}17 \\
(20.0 \%)\end{array}$ & $\begin{array}{l}14 \\
(16.5 \%)\end{array}$ & \\
\hline $\begin{array}{l}\text { Biopsy Gleason score, median } \\
\text { (IQR) }\end{array}$ & $6(5,8)$ & $7(6,8)$ & 0.020 & $\begin{array}{l}7(5.75 \\
8)\end{array}$ & $7(6,8)$ & 0.214 \\
\hline High risk ${ }^{\square} \mathrm{n}(\%)$ & $62(45.3 \%)$ & $\begin{array}{l}53 \\
(55.8 \%)\end{array}$ & 0.115 & $\begin{array}{l}43 \\
(50.6 \%)\end{array}$ & $\begin{array}{l}46 \\
(54.1 \%)\end{array}$ & 0.645 \\
\hline
\end{tabular}

SD: standard deviation; BMI: body mass index; ASA: American Society of Anesthesiologists; IIEF: International Index of Erectile Function; IQR: inter-quartile range.

according to the D'Amico risk classifications

Table 2 delineated the perioperative and pathological outcomes in detail. Within the matched settings, no surgery was converted to an open approach in either arm. Patients in the RARP group had a significantly 
shorter mean OT than those in the LRP group (146.0 vs. 167.9 min, $p<0.001)$, but no significant difference in the mean EBL was found between the two groups (152.6 vs. $166.4 \mathrm{~mL}, p=0.200$ ). 55 $(64.7 \%)$ and $50(58.8 \%)$ cases underwent ePLND in the RARP and LRP groups, respectively $(p=0.430)$, while nerve sparing technique was more frequently completed in patients undergoing RARP than those with LRP $(48.2 \%$ vs. $32.9 \%, p=0.042)$. There were no significant differences in the probability of transfusion and $>$ Grade II postoperative complications between the two groups ( $p=0.192$ and $p=1.000$, respectively). The distributions of pathologic T2 and T3 disease were comparable among the RARP and LRP groups $(p=0.345)$, and the comparability between the two groups remained with regard to the median specimen Gleason score and hospital stay length ( $p=0.179$ and $p=0.563$, respectively). The occurrence rates of PSM and positive lymph node were also statistically similar between the RARP and LRP arms ( $p=0.260$ and $p=0.501$, respectively). However, patients in the LRP group had a tendency towards a higher incidence of $\leq$ Grade II complications than those in the RARP group $(p=0.036)$.

Table 2

Perioperative outcomes for RARP and LRP after propensity score matching.

\begin{tabular}{|c|c|c|c|}
\hline Variable & $\operatorname{RARP}(n=85)$ & $\operatorname{LRP}(n=85)$ & $p$ value \\
\hline Operative time, min, mean (SD) & $146.0(40.4)$ & $167.9(34.2)$ & $<0.001$ \\
\hline Estimated blood loss, $\mathrm{mL}$, mean (SD) & $152.6(60.5)$ & $166.4(78.2)$ & 0.200 \\
\hline ePLND, n (\%) & $55(64.7 \%)$ & $50(58.8 \%)$ & 0.430 \\
\hline Nerve sparing procedures, n (\%) & $41(48.2 \%)$ & $28(32.9 \%)$ & 0.042 \\
\hline Open conversion, n (\%) & $0(0 \%)$ & $0(0 \%)$ & - \\
\hline Transfusion, n (\%) & $3(3.5 \%)$ & $7(8.2 \%)$ & 0.192 \\
\hline \multicolumn{4}{|l|}{ Postoperative pathology } \\
\hline Pathological T stage, n (\%) & & & 0.345 \\
\hline pT2 & $49(57.6 \%)$ & $55(64.7 \%)$ & \\
\hline pT3 & $36(42.4 \%)$ & $30(35.3 \%)$ & \\
\hline Specimen Gleason score, median (IQR) & $7(5.5,8)$ & $7(6,8)$ & 0.179 \\
\hline Positive surgical margin, $\mathrm{n}(\%)$ & $15(17.6 \%)$ & $21(24.7 \%)$ & 0.260 \\
\hline Positive lymph nodes, n (\%) & $13(15.3 \%)$ & $10(11.8 \%)$ & 0.501 \\
\hline Postoperative complications, n (\%) & $8(9.4 \%)$ & $18(21.2 \%)$ & 0.033 \\
\hline$\leq$ Grade II complications & $6(7.1 \%)$ & $15(17.6 \%)$ & 0.036 \\
\hline$>$ Grade II complications & $2(2.4 \%)$ & $3(3.5 \%)$ & 1.000 \\
\hline Hospital stay, days, median (IQR) & $14(14,15)$ & $15(14,15)$ & 0.563 \\
\hline
\end{tabular}


ePLND: extended pelvic lymph nodes dissection; SD: standard deviation; IQR: inter-quartile range. Within the matched cohort, median follow-up durations after RARP and LRP were 29 and 23 months, respectively. As shown in Table 3, no significant differences were detected between the two groups in terms of the mean total serum PSA at postoperative 6 months and last follow-up ( $p=546$ and $p=0.688$, respectively). The appearances of BCR have happened in 8 and 11 patients following RARP and LRP, respectively, over the periods covered by the follow-ups. The likelihoods of BCR-free survivals of immediate- and high-risk patients following RARP and LRP were also statistically similar after matching $(p=0.228)$ (Figure. 1).

Table 3

Postoperative outcomes for RARP and LRP after propensity score matching.

\begin{tabular}{|llll|}
\hline Variable & RARP $(\boldsymbol{n}=\mathbf{8 5})$ & LRP $(\boldsymbol{n}=\mathbf{8 5})$ & $\boldsymbol{p}$ value \\
\hline Oncology: postoperative total PSA, ng/mL & & & \\
\hline Postoperative 6 months, mean (SD) & $0.053(0.062)$ & $0.047(0.078)$ & 0.546 \\
\hline Last follow-up, mean (SD) & $0.218(0.955)$ & $0.280(1.021)$ & 0.688 \\
\hline Urinary continence & & & \\
\hline Continent on removal of catheter, $\mathrm{n}(\%)$ & $33(38.8 \%)$ & $20(23.5 \%)$ & 0.031 \\
\hline Continent at 6 months, $\mathrm{n}(\%)$ & $66(77.6 \%)$ & $54(63.5 \%)$ & 0.043 \\
\hline Continent at last follow-up, $\mathrm{n}(\%)$ & $80(94.1 \%)$ & $72(84.7 \%)$ & 0.046 \\
\hline Erectile function & & & \\
\hline IIEF-5 score at postoperative 6 month, median (IQR) & $14(12,16)$ & $12(11,15)$ & 0.013 \\
\hline IIEF-5 score at last follow-up, median (IQR) & $14(11,16)$ & $12(11,14)$ & 0.009 \\
\hline
\end{tabular}

PSA: prostate specific antigen; SD: standard deviation; IIEF: International Index of Erectile Function; IQR: inter-quartile range.

The Foley catheter was routinely removed at postoperative 2 weeks regardless of the surgical approach. As described in Table 3, the proportions of patients reporting UC recovery at the moment of removing catheter (38.8\% vs. $23.5 \%, p=0.031)$, postoperative 6 months ( $77.6 \%$ vs. $63.5 \%, p=0.043)$, and last follow-up ( $94.1 \%$ vs. $84.7 \%, p=0.046)$ in the RARP group were significantly higher than that in the LRP group. Intriguingly, the difference in postoperative UC recovery obtained following RARP and LRP was gradually alleviated and close to be out of statistical significance. The patients in the RARP group achieved a significantly higher cumulative proportion of postoperative return to UC than those following $\operatorname{LRP}(p=0.011)$ (Figure. 2).

As presented in Table 3, within the matched cohort, significant differences were also revealed between the RARP and LRP groups with respect to the median IIEF- 5 score at postoperative 6 months and last follow- 
up ( $p=0.013$ and $p=0.009$, respectively), exhibiting the superiority of RARP over LRP in erectile functional protection for men with immediate- and high-risk PCa.

\section{Discussion}

Given the limited overall survival benefits and considerable adverse events after RP for D'Amico low-risk $\mathrm{PCa}$, the role of RP in managing D'Amico low-risk PCa remains highly contentious $(3,4)$. While RP for D'Amico immediate- and high-risk PCa could achieve favorable survival benefits from preventing further metastatic seeding of potentially lethal clones of PCa cells (9). In 2020, RP is widely perceived as appropriate for men with intermediate-risk and high-risk PCa rather than those bearing D'Amico low-risk PCa (9). Although RARP has been widely diffused for surgically handling localized PCa, the paucity of high-level evidence still triggers the controversy on the impacts of RARP and LRP on oncological and functional outcomes obtained after surgery. What's worse, there has been no study which was immersed in comparing RARP and LRP for intermediate-risk and high-risk PCa up to now, while the cogent evidence concerning the functional and oncological efficacy of RARP and LRP for immediate- and high-risk localized PCa is of great clinical importance.

In this content, we designed this retrospective study which is the first one comparing RARP and LRP for immediate- and high-risk PCa. To eliminate the influences of any significant differences in any preoperative factors and selection bias, we rigorously utilized the PM method to guarantee the similarity in all preoperative parameters between the two groups. Within the well-balanced matched cohort, our results revealed the superiority of RARP in functional preservation coupled with fewer postoperative $\leq$ Grade II complications than LRP without cancer control being compromised for managing immediateand high-risk PCa.

As regards the extended mean OT in the LRP group, this significant difference may be attributable to the fact that the robotic platform facilitates suturing, one of the most challenging procedures during the standard laparoscopic approach (20), and this advantage is even more obvious when comparing RARP and LRP for the immediate- and high-risk patients enrolled in our analysis. As to the similar mean EBL and transfusion rates following RARP and LRP, the similarity can be explained by the counterbalance between the contributing factors including the better visualization, improved dexterity, and higher precision to minimize bleeding during RARP and unfavorable factors leading to EBL including more ePLNDs and nerve sparing procedures done in the RARP group. Both Johnson et al. (21) and Papachristos et al. (22) also achieved similar outcomes regarding EBL and OT after RARP and LRP to ours, in spite of the drastic variation of mean EBL and OT offered from different medical centers. The variation could be easily interpreted when taking the surgeons' experience and patients' and tumors' characteristics into account.

Although both LRP and RARP are minimally invasive, the better visualization and higher precision offered by the robotic platform could help in further reducing the operative invasiveness and the hazard of organ injures $(20,21)$, which may translate into the significantly lower proportion of overall and $\leq$ Grade II 
postoperative complications after RARP in our analysis. However, these advantages mentioned above may be restricted by the higher rate of ePLNDs underwent in the RARP group which was associated with the occurrences of symptomatic lymphocele, the most frequent > Grade II complication in our study, thus resulting in similar rates of $>$ Grade II postoperative complications. The comparability of the incidence rates of postoperative > Grade II complications between the two groups in our analysis was consistent with those reported in the contemporary series $(14,20-24)$ comparing RARP and LRP, demonstrating the similar operative safety of RARP and LRP for immediate- and high-risk PCa in experienced hands.

Surgical approach to RP should be tempered with critical significance of cancer control, especially when managing immediate- and high-risk PCa. Consistent with the results reported in published analyses (13, $14,25)$, no significant difference in PSM rate was discovered in our study comparing RARP $(17.6 \%)$ and LRP (24.7\%) for immediate- and high-risk PCa. Evaluated BMI and large prostate volume were considered as the independent predictors of PSMs in men with organ-confined PCa (26), and cumulative evidences revealed that margin status following RP was related to surgical experience $(26,27)$. Fortunately, all these influencing elements were under stringent control with PM method being employed in this single-center analysis, thus greatly contributing to the similarity in PSM rates after RARP and LRP. Of note, compared with the $15 \%$ mean rate of PSMs in RARP series including more than 100 cases (26), $17.6 \%$ rate of PSMs acquired after RARP in our analysis was relatively high even in highly experienced hands when removing immediate- and high-risk PCa, coordinating the caution that the more extensive the cancer, the higher the possibility of positive margins (26). Although PSMs in RP specimens were in consistent correction with an enhanced risk of PSA relapse $(28,29)$, the long-term impacts of PSMs on more robust clinical endpoints of the disease is variable and mostly depends on other variables, such as Gleason score, pathologic stage, and preoperative PSA $(16,30)$. Intriguingly, most of these decisive factors including Gleason score and preoperative PSA were the basis of D'Amico risk classifications, namely, the clinical endpoints, such as clinical recurrence rates, largely relied on preoperative baseline characteristics rather than PSMs after RP. In alignment with other series $(14,25)$ comparing RARP and LRP, the similarity in the BCR-free survivals obtained after RARP and LRP was still existing, corroborating the equivalent potency of the two procedures in cancer control even when managing immediate- and high-risk PCa.

The aims of RP are to completely eradicate localized PCa whilst, whenever possible, preserves UC and erectile function, namely, a trifecta outcome (4). Urinary incontinence after RP is one of the most adverse events that negatively impact patients' quality of life $(20,31)$. Multiple pathophysiologic mechanisms contribute to the emergence of post-prostatectomy incontinence (PPI). In addition to the biological/preoperative parameters encompassing the patient age at time of surgery, pre-existing lower urinary tract symptoms, high $\mathrm{BMl}$, and abnormal bladder function, the impairments of the integrity of anatomic supporting structures and neural components during the RP procedure appear to be crucial contributing factors to the development of PPI $(31,32)$. In our analysis, preoperative/biological parameters are comparable between the RARP and LRP groups with PM method being applied, but the robotic platforms allowed better preservation of membranous urethra and nerve branches and reconstruction of bladder neck, thus supporting the higher UC probability after RARP over the whole follow-up period. In the prospective randomized controlled study reported by Porpiglia et al. (25), UC rate 
after RARP was also significantly higher than that after LRP for localized PCa over the 5-year follow-up period. Our results also corroborated the outcomes obtained in the first multicenter, randomized, patientblinded controlled trial (LAP-01) (14) which demonstrated the improved postoperative return to UC of RARP over LRP. The advantage of robotic platforms in better surgical vision and higher precision for preserving the neurovascular structures could be greatly responsible for the superior erectile function recovery after RARP than that obtained after LRP.

Notably, several limitations should be taken into consideration when interpreting our conclusions.

Structural shortages in data collection were inevitable in a retrospective setting of our analysis. The study population, although well-balanced between the two groups, is relatively small. The long-term oncological survivals and functional recoveries could not be further evaluated over the relatively limited follow-up lengths. Certain complications may be undervalued, especially $\leq$ Grade II complications, in spite of the elaborative investigation of medical records and telephone interview.

Despite these limitations, our study is the first one designed to assess the perioperative, functional, and oncological outcomes acquired after RARP and LRP for localized immediate- and high-risk PCa up to now, and our conclusions are drew and strengthened on the basis of the comparability of all perioperative elements between the two arms and rigorous methodology.

\section{Conclusions}

For surgically managing immediate- and high-risk localized PCa, RARP tended to a lower risk of $\leq$ Grade II complications and superior functional preservation without cancer control being compromised when comparing with LRP. Our present conclusions need to be confirmed further on the basis of prospectively randomized trials with large sample sizes and sufficiently long follow-ups.

\section{Abbreviations}

RARP robot-assisted radical prostatectomy;

RP:radical prostatectomy;

LRP laparoscopic radical prostatectomy;

PCa:prostate cancer;

PPI post-prostatectomy incontinence;

PSA:prostate specific antigen;

PM propensity-score matching;

RCT:randomized controlled trial; 
PLND Pelvic lymph node dissection;

BMl:body mass index;

ASA American Society of Anesthesiologists;

OT:operative time;

IIEF International Index of Erectile Function;

EBL:estimated blood loss;

PSM positive surgical margin;

UC:urinary continence;

BCR biochemical recurrence.

\section{Declarations}

\section{Ethics approval and consent to participate}

Not applicable.

\section{Consent for publication}

Not applicable.

\section{Availability of data and materials}

The datasets used and/or analysed during the current study are available from the corresponding author on reasonable request.

\section{Competing interests}

The authors declare that they have no competing interests.

\section{Funding}

Not applicable.

\section{Authors' contributions}

All the authors contributed in the preparation of this work. DW, ZC, JH and LYL were drafted and revised the article, DW, LXQ and CLY were responsible for the theme, final editing, and preparation of the manuscript for submission, LWP, ZXC, ST, WGX and FB critically revised the manuscript. All authors read and approved the final manuscript. 


\section{Acknowledgements}

Not applicable.

\section{Author details}

${ }^{1}$ Department of Urology, the First Affiliated Hospital of Nanchang University, Yongwai street 17, Nanchang City, Jiangxi Province, China, ${ }^{2}$ Jiangxi Institute of Urology, Yongwai street 17, Nanchang City, Jiangxi Province, China.

\section{References}

1. Bray F, Ferlay J, Soerjomataram I, Siegel RL, Torre LA, Jemal A. Global cancer statistics 2018: GLOBOCAN estimates of incidence and mortality worldwide for 36 cancers in 185 countries. CA: a cancer journal for clinicians. 2018 Nov;68(6):394-424.

2. Ilic D, Djulbegovic M, Jung JH, Hwang EC, Zhou Q, Cleves A, et al. Prostate cancer screening with prostate-specific antigen (PSA) test: a systematic review and meta-analysis. BMJ (Clinical research ed). 2018 Sep 5;362:k3519.

3. Moschini M, Carroll PR, Eggener SE, Epstein JI, Graefen M, Montironi R, et al. Low-risk Prostate Cancer: Identification, Management, and Outcomes. European urology. 2017 Aug;72(2):238 - 49.

4. Mottet N, van den Bergh RCN, Briers E, Van den Broeck T, Cumberbatch MG, De Santis M, et al. EAUEANM-ESTRO-ESUR-SIOG Guidelines on Prostate Cancer-2020 Update. Part 1: Screening, Diagnosis, and Local Treatment with Curative Intent. European urology. 2021 Feb;79(2):243-62.

5. Tienza A, Akin Y, Rassweiler J, Gözen AS. A match-pair analysis of continence in intermediate and high-risk prostate cancer patients after robot-assisted radical prostatectomy: the role of urine loss ratio and predictive analysis. Prostate international. 2018 Sep;6(3):94-8.

6. Bill-Axelson A, Holmberg L, Garmo H, Rider JR, Taari K, Busch C, et al. Radical prostatectomy or watchful waiting in early prostate cancer. The New England journal of medicine. $2014 \mathrm{Mar}$ 6;370(10):932 - 42.

7. Wilt TJ, Jones KM, Barry MJ, Andriole GL, Culkin D, Wheeler T, et al. Follow-up of Prostatectomy versus Observation for Early Prostate Cancer. The New England journal of medicine. $2017 \mathrm{Jul}$ 13;377(2):132-42.

8. Chang AJ, Autio KA, Roach M 3rd, Scher HI. High-risk prostate cancer-classification and therapy. Nature reviews Clinical oncology. 2014 Jun;11(6):308-23.

9. Costello AJ. Considering the role of radical prostatectomy in 21 st century prostate cancer care. Nature reviews Urology. 2020 Mar;17(3):177-88.

10. Okegawa T, Omura S, Samejima M, Ninomiya N, Taguchi S, Nakamura Y, et al. Laparoscopic radical prostatectomy versus robot-assisted radical prostatectomy: comparison of oncological outcomes at a single center. Prostate international. 2020 Mar;8(1):16-21. 
11. Coughlin GD, Yaxley JW, Chambers SK, Occhipinti S, Samaratunga H, Zajdlewicz L, et al. Robotassisted laparoscopic prostatectomy versus open radical retropubic prostatectomy: 24-month outcomes from a randomised controlled study. The Lancet Oncology. 2018 Aug;19(8):1051-60.

12. Nossiter J, Sujenthiran A, Charman SC, Cathcart PJ, Aggarwal A, Payne H, et al. Robot-assisted radical prostatectomy vs laparoscopic and open retropubic radical prostatectomy: functional outcomes 18 months after diagnosis from a national cohort study in England. British journal of cancer. 2018 Feb 20;118(4):489-94.

13. Asimakopoulos AD, Pereira Fraga CT, Annino F, Pasqualetti P, Calado AA, Mugnier C. Randomized comparison between laparoscopic and robot-assisted nerve-sparing radical prostatectomy. J Sex Med. 2011 May;8(5):1503-12.

14. Stolzenburg JU, Holze S, Neuhaus P, Kyriazis I, Do HM, Dietel A, et al. Robotic-assisted Versus Laparoscopic Surgery: Outcomes from the First Multicentre, Randomised, Patient-blinded Controlled Trial in Radical Prostatectomy (LAP-01). European urology. 2021 Feb 8.

15. Porpiglia F, Morra I, Lucci Chiarissi M, Manfredi M, Mele F, Grande S, et al. Randomised controlled trial comparing laparoscopic and robot-assisted radical prostatectomy. European urology. 2013 Apr;63(4):606-14.

16. Umari P, Eden C, Cahill D, Rizzo M, Eden D, Sooriakumaran P. Retzius-Sparing versus Standard RobotAssisted Radical Prostatectomy: A Comparative Prospective Study of Nearly 500 Patients. The Journal of urology. 2021 Mar;205(3):780-90.

17. Touijer AK, Guillonneau B. Laparoscopic radical prostatectomy. Urologic oncology. 2004 MarApr;22(2):133-8.

18. Rosen RC, Riley A, Wagner G, Osterloh IH, Kirkpatrick J, Mishra A. The international index of erectile function (IIEF): a multidimensional scale for assessment of erectile dysfunction. Urology. 1997 Jun;49(6):822-30.

19. Dindo D, Demartines N, Clavien PA. Classification of surgical complications: a new proposal with evaluation in a cohort of 6336 patients and results of a survey. Annals of surgery. 2004 Aug;240(2):205-13.

20. Carbonara U, Srinath M, Crocerossa F, Ferro M, Cantiello F, Lucarelli G, et al. Robot-assisted radical prostatectomy versus standard laparoscopic radical prostatectomy: an evidence-based analysis of comparative outcomes. World journal of urology. 2021 Apr 11.

21. Johnson I, Ottosson F, Diep LM, Berg RE, Hoff JR, Wessel N, et al. Switching from laparoscopic radical prostatectomy to robot assisted laparoscopic prostatectomy: comparing oncological outcomes and complications. Scandinavian journal of urology. 2018 Apr;52(2):116-21.

22. Papachristos A, Basto M, Te Marvelde L, Moon D. Laparoscopic versus robotic-assisted radical prostatectomy: an Australian single-surgeon series. ANZ J Surg. 2015 Mar;85(3):154-8.

23. Hakimi AA, Blitstein J, Feder M, Shapiro E, Ghavamian R. Direct comparison of surgical and functional outcomes of robotic-assisted versus pure laparoscopic radical prostatectomy: singlesurgeon experience. Urology. 2009 Jan;73(1):119-23. 
24. Menon M, Shrivastava A, Tewari A, Sarle R, Hemal A, Peabody JO, et al. Laparoscopic and robot assisted radical prostatectomy: establishment of a structured program and preliminary analysis of outcomes. The Journal of urology. 2002 Sep;168(3):945-9.

25. Porpiglia F, Fiori C, Bertolo R, Manfredi M, Mele F, Checcucci E, et al. Five-year Outcomes for a Prospective Randomised Controlled Trial Comparing Laparoscopic and Robot-assisted Radical Prostatectomy. European urology focus. 2018 Jan;4(1):80-6.

26. Yossepowitch O, Briganti A, Eastham JA, Epstein J, Graefen M, Montironi R, et al. Positive surgical margins after radical prostatectomy: a systematic review and contemporary update. European urology. 2014 Feb;65(2):303-13.

27. Bravi CA, Tin A, Vertosick E, Mazzone E, Martini A, Dell'Oglio P, et al. The Impact of Experience on the Risk of Surgical Margins and Biochemical Recurrence after Robot-Assisted Radical Prostatectomy: A Learning Curve Study. The Journal of urology. 2019 Jul;202(1):108-13.

28. Jo JK, Hong SK, Byun SS, Zargar H, Autorino R, Lee SE. Positive surgical margin in robot-assisted radical prostatectomy: correlation with pathology findings and risk of biochemical recurrence. Minerva urologica e nefrologica $=$ The Italian journal of urology and nephrology. 2017 Oct;69(5):493-500.

29. Zhang L, Wu B, Zha Z, Zhao H, Jiang Y, Yuan J. Positive surgical margin is associated with biochemical recurrence risk following radical prostatectomy: a meta-analysis from high-quality retrospective cohort studies. World journal of surgical oncology. 2018 Jul 3;16(1):124.

30. Checcucci E, Veccia A, Fiori C, Amparore D, Manfredi M, Di Dio M, et al. Retzius-sparing robot-assisted radical prostatectomy vs the standard approach: a systematic review and analysis of comparative outcomes. BJU Int. 2020 Jan;125(1):8-16.

31. Asimakopoulos AD, Topazio L, De Angelis M, Agrò EF, Pastore AL, Fuschi A, et al. Retzius-sparing versus standard robot-assisted radical prostatectomy: a prospective randomized comparison on immediate continence rates. Surgical endoscopy. 2019 Jul;33(7):2187-96.

32. Heesakkers J, Farag F, Bauer RM, Sandhu J, De Ridder D, Stenzl A. Pathophysiology and Contributing Factors in Postprostatectomy Incontinence: A Review. European urology. 2017 Jun;71(6):936-44.

\section{Figures}




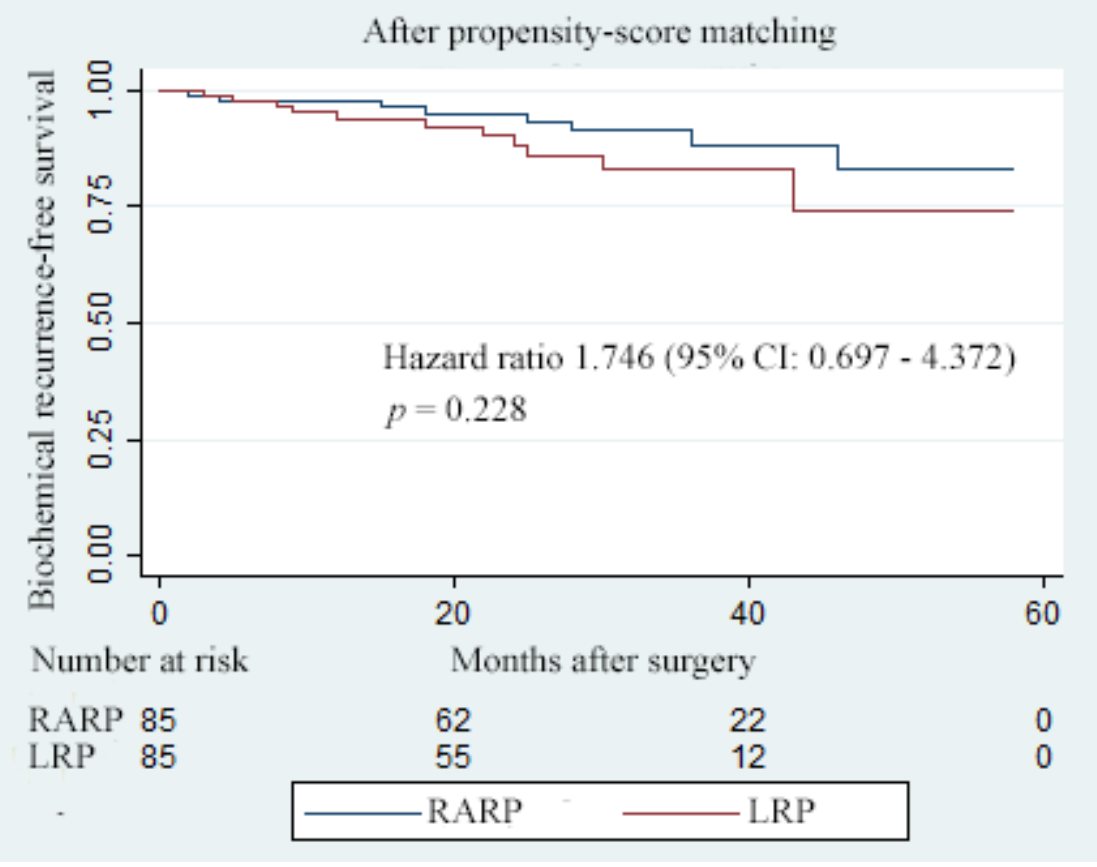

\section{Figure 1}

Kaplan-Meier curves showing biochemical recurrence-free survival for patients undergoing robotassisted and laparoscopic radical prostatectomy over the follow-up durations.

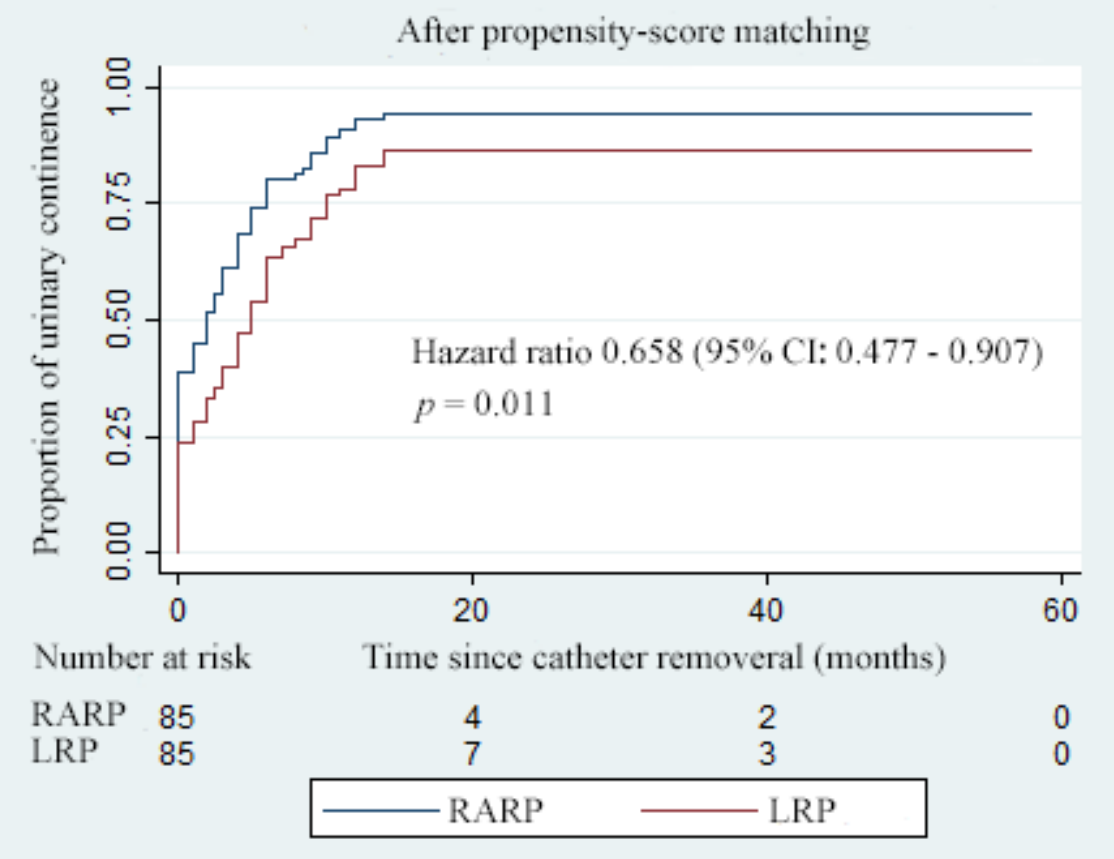

\section{Figure 2}

Kaplan-Meier curves showing the proportion of urinary continence (UC) in patients undergoing robotassisted and laparoscopic radical prostatectomy over the follow-up durations. UC was defined as requiring no pad or preventively using one dry pad per day. 